Original article

\title{
Consensus in percutaneous bunionette correction
}

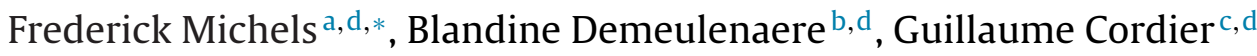 \\ a Orthopaedic Department, AZ Groeninge, 8500 Kortrijk, Belgium \\ b Clinique Blomet, institut de la cheville et du pied, 136, bis rue Blomet, 75005 Paris, France \\ c Orthopaedic Department, Mérignac sports clinic, 2, rue Georges-Negrevergne, 33700 Mérignac, France \\ ${ }^{\mathrm{d}}$ MIFAS by GRECMIP (Minimally Invasive Foot and Ankle Society), Mérignac, France
}

\section{A R T I C L E I N F O}

\section{Article history:}

Received 5 November 2019

Accepted 25 March 2020

Available online xxx

\section{Keywords:}

Bunionette

Tailor's bunion

Percutaneous surgery

Minimally invasive surgery

Distal metatarsal metaphyseal osteotomy

DMMO

French

\begin{abstract}
A B S T R A C T
Purpose: The percutaneous treatment of bunionette deformity has been demonstrated as a reliable and satisfying technique with low risk of complications. However, there are some obvious variations in the surgical technique and perioperative protocol. The purpose of this study is to analyze the currently used techniques and to look for some agreements.

Hypothesis: There are some points of agreement in surgical technique and perioperative protocol when using a percutaneous technique to treat bunionette deformity.

Methods: A survey was sent to 50 orthopedic surgeons with specific experience in percutaneous techniques. The questions were related to different aspects of the surgical bunionette procedure and the perioperative protocol.

Results: A response rate of $92.0 \%$ was obtained. Several points of agreement were found. A condylectomy is rarely used while an osteotomy is performed in almost all procedures. This osteotomy is single (95.7\%), complete (66.2-72.7\%) and performed with a Shannon long burr (73.9\%). The location of the osteotomy depends of the deformity (63.0\%).

Discussion: This study demonstrates some consensus in the use of the surgical technique and the perioperative protocol. The percutaneous oblique osteotomy is the preferred technique while a condylectomy is only rarely used.

Level of evidence: $\mathrm{V}$, Survey study.
\end{abstract}

(c) 2020 Elsevier Masson SAS. All rights reserved.

\section{Introduction}

A bunionette deformity, or tailor's bunion, is a painful prominence on the lateral aspect of the fifth metatarsal head. Surgical treatment can be considered if conservative treatment has failed to relieve the symptoms. There are many different surgical techniques to treat this deformity. In recent years, there has been a move towards the development and use of minimally invasive and percutaneous surgical techniques $[1,2]$.

The first percutaneous techniques have been described by De Prado and Isham [3]. Further studies, assessing the clinical results, found similar results as the open techniques but a lower risk of complications [4-6]. Especially the hardware free percutaneous technique offers this major advantage as complications are very often related to hardware [7]. The advent of these new techniques offers new opportunities, but several questions remain

\footnotetext{
* Corresponding author.

E-mail address: frederick_michels@hotmail.com (F. Michels).
}

unanswered. Between the percutaneous techniques some variations have been described: condylectomy or not, double or single osteotomy, complete or incomplete osteotomy, location of the osteotomy [3-6,8,9]. There are also some differences in the perioperative protocol. This can raise some confusion about certain points e.g. use of tourniquet, use of antibiotics, use of vitamin C, physiotherapy. Many of these questions are difficult to answer with randomized studies. For this reason, the opinion of a group of experts is useful.

The purpose of this study is to formulate an answer on the following main question: can we find some points of agreement in surgical technique and perioperative protocol when using a percutaneous technique to treat bunionette deformity? For this reason we performed a survey study in a group of orthopedic surgeons with a specific experience in percutaneous surgery. The answers of this study can provide some interesting information for surgeons willing to start with this technique. The results are also interesting for the more experienced surgeons who want to compare their own habits to a larger group of experienced surgeons. 


\section{Materials and methods}

A survey was send to 50 orthopedic surgeons with a specific experience in the use of percutaneous techniques to treat forefoot problems.

Inclusion criteria were that the surgeons must have been trained in foot and ankle surgery. They should have an extensive clinical and didactic experience with the use of percutaneous techniques. Only surgeons with at least 5 years' experience with percutaneous surgery in general and the percutaneous hardware free technique in particular were included. All surgeons were member of the MIFAS (Minimally Invasive Foot and Ankle Society, a new name for the GRECMIP (Group of Research and Study in Minimally Invasive Surgery of the Foot). This is an international group of orthopedic surgeons with a special interest in research about minimally invasive foot and ankle surgery. The questionnaire was designed to obtain data about the surgical technique and the perioperative protocol. A cover letter was included, explaining the purpose of the survey. No ethical issues were raised, and no conflicts of interests were registered concerning this study.

\section{Results}

Response was obtained from 46 of the 50 invited surgeons which corresponds with an overall response rate of $92.0 \%$. The participants had an average of $10.6 \pm 2.9$ years (range: $5-16$ ) experience with the percutaneous techniques in forefoot correction. In addition, the responding surgeons are from 17 different countries and all of them have an experience in teaching percutaneous techniques. The participating countries are: France (20), UK (4), Morocco (3), Spain (2), Mexico (2), Switzerland (2), Germany (2), Belgium (2), Argentina (1), Romania (1), Brazil (1), Japan (1), Poland (1), Portugal (1), Italy (1), Lebanon (1), Austria (1). The answers on the different questions are reported below and in Table 1 .

\subsection{Perioperative items}

The majority of the surgeons $(80.4 \%, 37 / 46)$ treats the patients in one day clinic if the procedure is not associated with more invasive techniques. Different anesthesiologic techniques are used.

There was no consensus about the use of vitamin $C$ in the prevention of complex regional pain syndrome. In our survey it was more commonly used among the French surgeons. Peroperative antibiotics were used in $58.7 \%$. In contrast, the French surgeons were using less frequently antibiotics because no hardware was left inside the patient. There was agreement to use a postoperative shoe and a reducing bandage. A minimal period of elevation varying from 1 to 3 weeks was recommended by $82.6 \%$ (38/46) of the surgeons. Physiotherapy was recommended by a smaller part of the surgeons $(23.9 \%, 11 / 46)$. The most common complication in bunionette surgery was delayed bony healing.

\subsection{Peroperative items}

Most surgeons (82.6\%, 38/46) didn't use a tourniquet. The other colleagues, using a tourniquet, did it for practical reasons, as it allowed to prepare all forefoot procedures in a similar way. Most surgeons $(89.1 \%, 41 / 46)$ used fluoroscopy. A mini image intensifier was used by $50 \%$ of the users. The mini image intensifier was the preferred tool. However, this was not (always) available in some hospitals. The fluoroscopy was used twice: to choose the position burr before the osteotomy $(53.6 \%, 22 / 41)$ and to check the result of the procedure after the osteotomy $(100 \%, 41 / 41)$.

Most surgeons $(78.2 \%, 36 / 46)$ agreed to perform this technique as a bilateral procedure if requested by the patient. Most surgeons
Table 1

Perioperative protocol percutaneous bunionette correction.

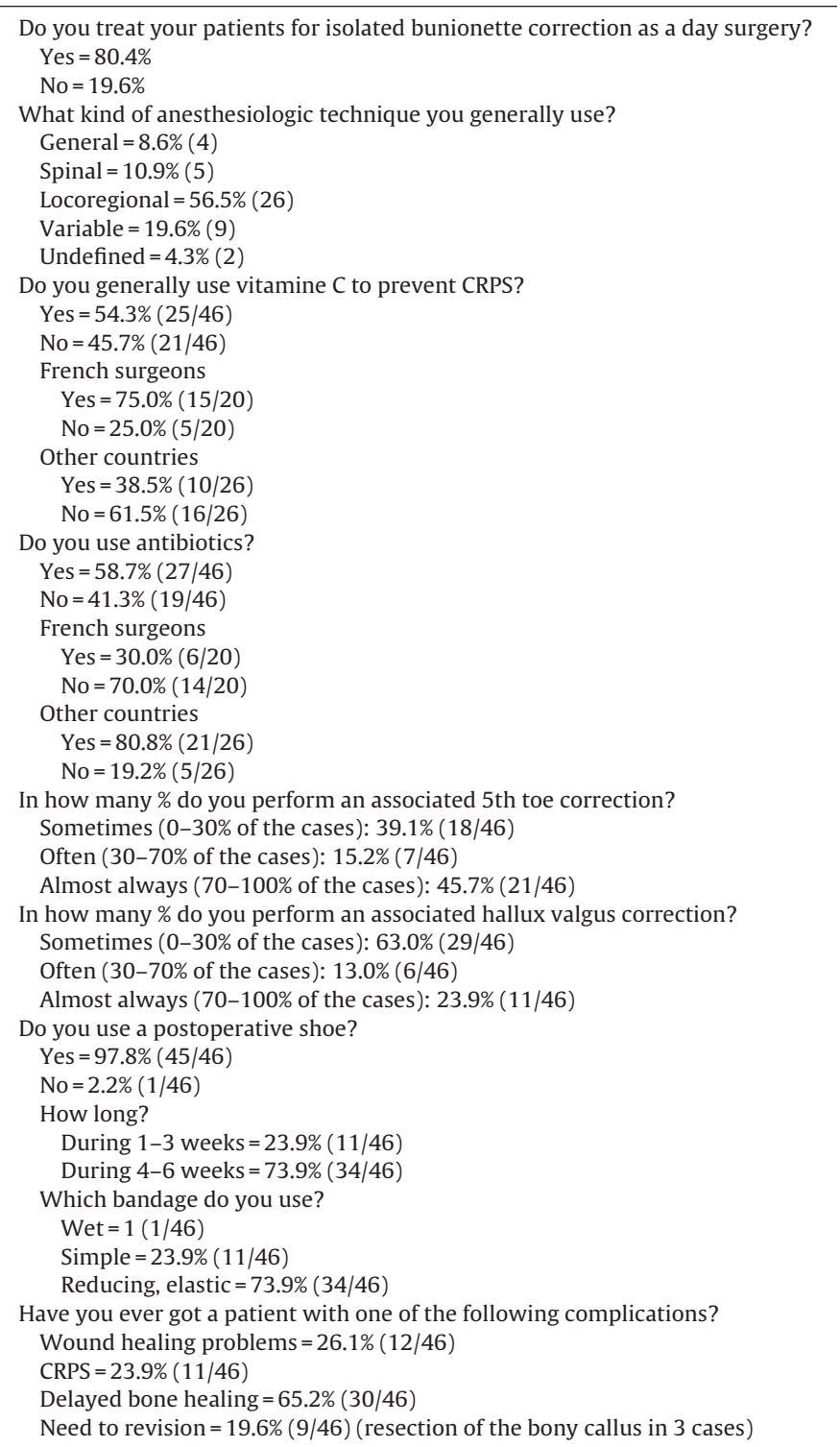

(89.1\%, 41/46) always use the percutaneous technique when a surgical treatment of bunionette is indicated. The others reported using an open technique in very selected cases: hardware removal, revision, osteoarthritis. An associated correction of the 5th toe or hallux valgus was performed in a variable degree depending of the surgeon.

\subsection{Items about surgical technique}

There's agreement (97.8\%) among the experts about not performing a condylectomy in every patient. Performing a condylectomy would be considered in $0-10 \%$ of the cases. There's agreement (91.3\%) to perform an osteotomy of the 5th metatarsal in $90-100 \%$ of the cases. Most surgeons $(65.2 \%, 30 / 46)$ preferred a complete osteotomy, while others preferred a partial osteotomy $(28.2 \%, 13 / 46)$, or both techniques $(6.5 \%, 3 / 46)$. Almost all $(95.7 \%)$ performed a single osteotomy. The location of the osteotomy depended for most surgeons on the site of the deformity $(63.0 \%$, $29 / 46$ ) while others routinely performed a distal osteotomy (37.0\%, 17/46). The preferred burrs to perform a bunionette procedure 

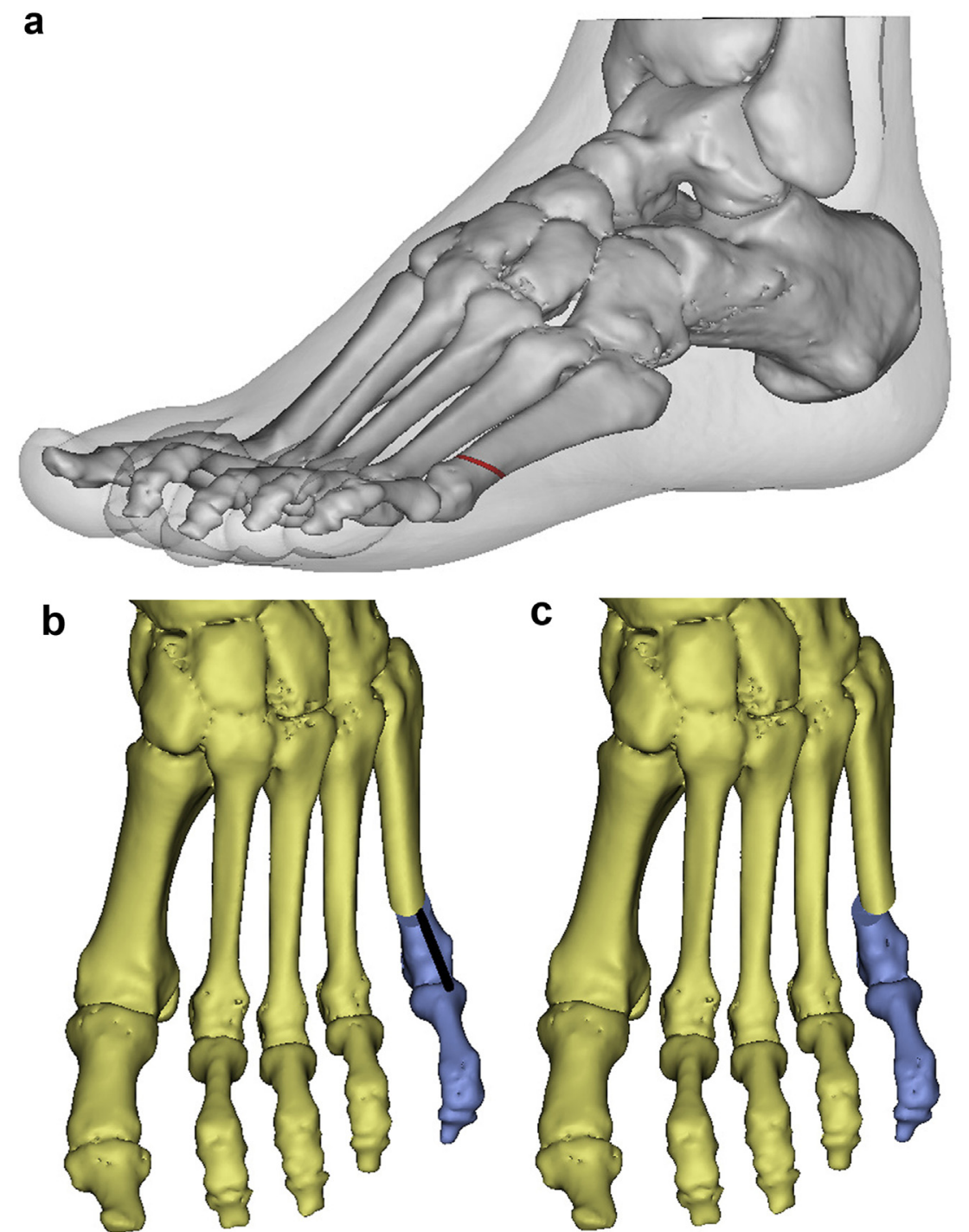

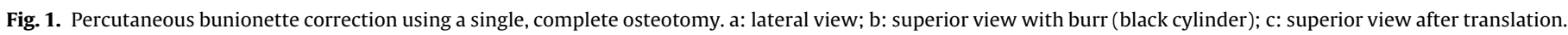

were: Shannon long burr $(73.9 \%, 34 / 46)$, Shannon Short burr $(23.9 \%$, $11 / 46)$, wedge burr $(2.2 \%, 1 / 46)$.

\section{Discussion}

The main finding of the present study was that there appear to be some points of agreement in the approach of patients when performing a percutaneous bunionette procedure.

In recent years there is an evolution from open techniques towards the use of minimally invasive and percutaneous techniques $[1,2]$. In bunionette surgery, the clinical outcome of the percutaneous technique is comparable to standard surgical procedures but both techniques have some specific benefits and drawbacks [10]. The conventional open surgical techniques are related to a higher risk of specific complications due to the open approach and the use of hardware: wound healing problems, infections and hardware problems [11,12]. Necas et al. reported 5 complications in 34 open bunionette corrections [12]. Three patients needed additional surgery due to complications: 1 infection, 1 screw migration, 1 transfer metatarsalgia. Hrubina et al. reported 3 complications in 23 open procedures: 1 delayed union, 1 superficial wound infection and a distal screw migration [11]. The main advantages of the percutaneous approach are due to the minimally invasive approach and the hardware free technique. The minimally invasive approach limits the risk to wound problems and infections. The hardware free technique avoids hardware problems. Besides the benefits of the percutaneous techniques there are also some risks. A delayed bone healing is the most common complication [4,5]. Michels et al. described 2 patients with hypertrophic callus in 21 percutaneous procedures due to delayed bone healing [5]. Laffenêtre et al. described 1 delayed bone healing in 49 cases [4]. The percutaneous approach offers only very limited visual control. For this reason it is needed to participate to training on sawbones and cadaveric specimens before using these techniques in clinical practice. As the learning curve for bunionette surgery is small, this technique can be one of the first percutaneous techniques to use in clinical practice [13].

In the publications of the recent years two groups of minimally invasive corrections can be distinguished: the mini-open techniques using a percutaneous K-wire [14-19] and the percutaneous techniques using no additional hardware [3-6,8-10,13,20]. Our study was limited to the surgeons using this second technique. In a recent review the results of both groups were compared [21]. A higher risk of complications was found in the group using the mini-open technique. Wound problems, infection and transfer metatarsalgia were the most encountered postoperative problems. A recent meta-analysis included 28 studies about surgical treat- 

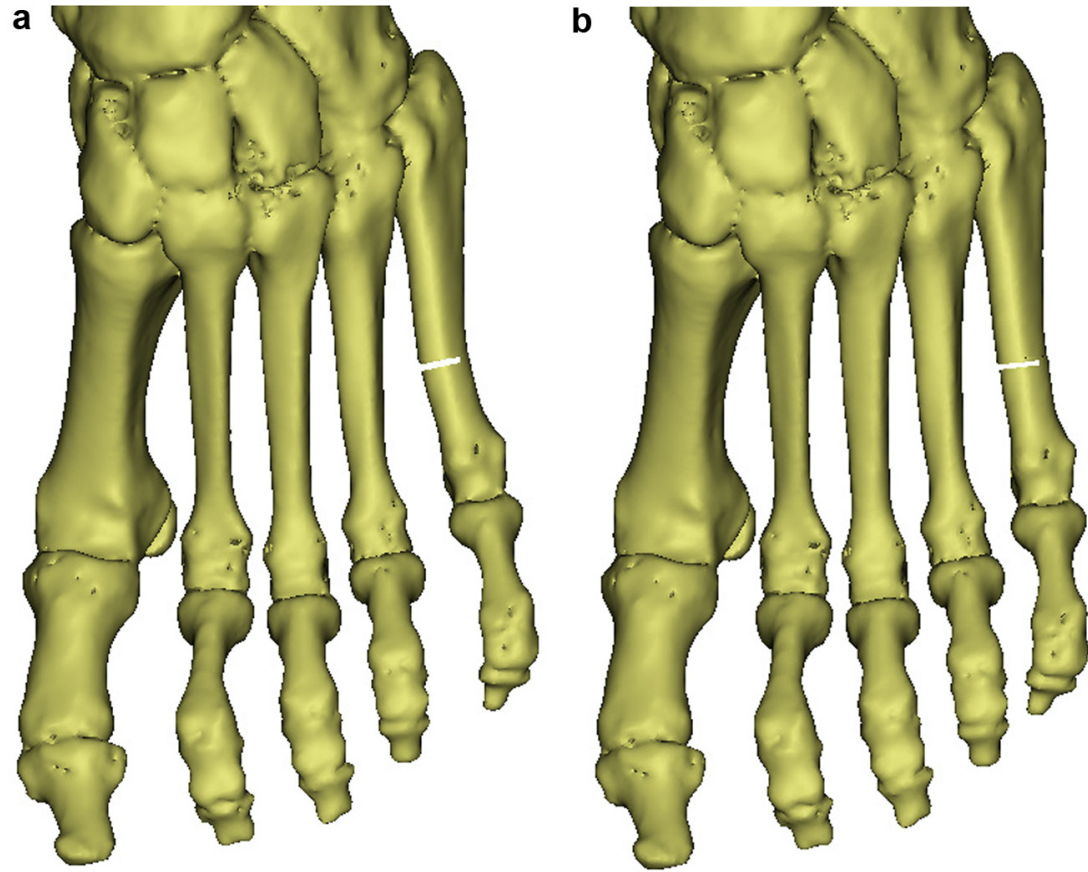

Fig. 2. Percutaneous bunionette correction using a single, partial osteotomy. a: superior view of incomplete osteotomy; b: superior view after rotation.

ment of bunionette deformity [7]. They demonstrated that more than $50 \%$ of the postoperative complications were related to the use of hardware. For the reasons described above, we excluded the mini-open techniques using hardware.

Our study found different points of agreement about the surgical techniques. The earlier descriptions of the percutaneous technique distinguish 2 steps: the condylectomy and the osteotomy $[3,5,8]$. Today, according to the findings in this study, a condylectomy is rarely used. As more recent publications describe similar good results with decreasing use or without the use of a condylectomy, there's probably a decrease of using this part of the technique $[4,6,22]$. This evolution can be explained by different reasons. First, as the fifth metatarsal head is quite small, the extravalue of this condylectomy may be very limited. Second, the condylectomy complicates the surgical technique because a certain part of bone needs to be removed. The removed part needs to a have certain volume to make a difference. On the other hand, one should avoid to remove too much bone from this small metatarsal head. Next, the mashed bone, produced by the resection, should be removed from the patient by the percutaneous approach. Finally, the condylectomy poses the patients at risk to more postoperative complications as inflammation close to the joint, damage of the joint and irritation by remaining bone fragments. For the reasons discussed above the authors recommend to use the condylectomy only in selected cases.

This study found some agreement about the use an osteotomy. An osteotomy of the fifth metatarsal is considered as the most important step (Fig. 1). The technique is performed with a long Shannon burr and controlled with a mini image intensifier. The single osteotomy was the preferred method. A double osteotomy, previously described by de Prado, is only used by few surgeons [3]. Most surgeons prefer a complete osteotomy, which is technically less demanding than a partial osteotomy (Fig. 2). Although an incomplete osteotomy may heal faster, it can be more difficult to obtain and keep an important translation of the metatarsal head. The location of the osteotomy is still a point of disagreement. Most surgeons adapt the location to the deformity while others routinely perform a distal osteotomy. A proximal osteotomy enables a larger degree of correction but is associated with a higher complication rate [7]. Bone complications, such as delayed union, nonunion, avascular necrosis, and fracture, occurred most often after proximal open osteotomies (9\%), although the clinical results did not significantly differ $[7,23]$. This may be due to an interruption of the blood supply as the nutrient artery enters the fifth metatarsal around the junction of the middle and proximal thirds of the diaphysis $[22,24]$. We should question if the limited benefits of a more proximal osteotomy outweigh the increased risk for complications.

This study offers some information useful for clinical practice. Besides the technical aspects of the surgical technique, also the perioperative procedure was assessed. Most of these aspects are difficult to prove with evidence based studies. The information in this study can guide surgeons in decision making for the different perioperative aspects when performing this procedure.

This study has some important limitations. A limitation due to the concept of a survey study is the fact that no direct results are measured. For this reason, we only included the opinion of surgeons with a fair amount of experience using this technique. The experienced surgeons may have adapted their own technique to their personal experiences. The fact that they are all instructors may help them to see other variations and offer the opportunity to discuss about their experiences. For this reason this study transcends the level of a single expert opinion.

Another limitation may be the disproportional inclusion of larger group of French surgeons. Because this technique has become popular in France since many years, more French surgeons met the inclusion criteria for the requested experience. If some bias was suspected, the results were separately assessed. However, the differences between the French and other surgeons were not very strict and were only limited to 2 items: the use of vitamin $C$ and the use of perioperative antibiotics.

\section{Conclusion}

This study presents several points of agreement among experts when performing a percutaneous bunionette correction. The earlier described condylectomy is only occasionally used. The 
percutaneous osteotomy of the fifth metatarsal is the most important procedure. The technique is performed with a long Shannon burr and controlled with a mini image intensifier. Most surgeons perform a single complete osteotomy. A postoperative shoe is routinely used during several weeks.

\section{Disclosure of interest}

The authors declare that they have no competing interest.

\section{Funding}

No funding has been received for this study.

\section{Contribution}

All authors participated in the study and the writing of the manuscript.

\section{Acknowledgement}

We thank the following surgeons who collaborated in this survey: Abi Raad Georges, Biz Carlo, Carvalho Paulo, Cermolacce Christophe, Cerrato Rebecca, Chomicki-Bindas Piotr, Cordier Guillaume, Darcel Veronique, Delmi Marino, Demeulenaere Blandine, Elkaïm Marc, Frey Solenne, Hochheuser Georg, Ismael Farid, Karrakchou Samir, Laffenêtre Olivier, Ribeiro Lara Luiz, Leidolf Hermann, Lucas y Hernandez Julien, Malagelada Francesc, Mattes Frank, Michels Frederick, Molinier François, Pellegrino Alexandro, Perera Anthony, Piclet Barbara, Rabat Eduard, Torrent Josep, Vernois Joël, Toshinori Kurashige, Jean-Yves Coillard, Jean Claude Moati, Remy Touzard, Marius Uscatu, Mickael Andrieu, Frederic Salmeron, Patrick Deprez, Javier Del Vecchio, Thami Tazi, Julien Beldame, Alberto Cuellar Avaroma, Ana Cristina King, Julien Laborde, Pierre Barouk, Emmanuel Richelme, Kartik Hariharan.

\section{References}

[1] Bauer T. Percutaneous forefoot surgery. Orthop Traumatol Surg Res 2014;100:S191-204.

[2] Redfern D, Vernois J, Legré BP. Percutaneous surgery of the forefoot. Clin Podiatr Med Surg 2015:32:291-332.

[3] De Prado M, Ripoll PL, Golano P. Juanete de sastre. In: Cirugia percutanea del pie. Tecnicas quirurgicas, indicaciones, bases anatomicas. 1st ed. Barcelona: Masson Elsevier; 2003. p. 129-48.
[4] Laffenêtre O, Millet-Barbé B, Darcel V, Lucas Y, Hernandez J, Chauveaux D. Percutaneous bunionette correction: results of a 49 -case retrospective study at a mean 34 months' follow-up. Orthop Traumatol Surg Res 2015;101:179-84.

[5] Michels F, Van Der Bauwhede J, Guillo S, Oosterlinck D, de Lavigne C. Percutaneous bunionette correction. Foot Ankle Surg 2013;19:9-14.

[6] Molenaers B, Vanlommel J, Deprez P. Percutaneous hardware free corrective osteotomy for bunionnette deformity. Acta Orthop Belg 2017;83:284-91.

[7] Martijn HA, Sierevelt IN, Wassink S, Nolte PA. Fifth metatarsal osteotomies for treatment of bunionette deformity: a meta-analysis of angle correction and clinical condition. J Foot Ankle Surg 2018;57:140-8.

[8] Michels F, Guillo S. GRECMIP. Tailor's bunionectomy. In: Saxena A, editor. International advances in foot and ankle, surgery. London, UK: Springer; 2012. p. 99-106.

[9] Teoh KH, Hariharan K. Minimally invasive distal metatarsal metaphyseal osteotomy (DMMO) of the fifth metatarsal for bunionette correction. Foot Ankle Int 2018;39:450-7.

[10] Morawe GA, Schmieschek MHT. Minimally invasive bunionette correction. Oper Orthop Traumatol 2018;30:184-94.

[11] Hrubina M, Skotak M, Letocha J, Dzupa V. The modified scarf osteotomy in the treatment of tailor's bunion: midterm follow-up. Acta Orthop Belg 2015;81:57-64.

[12] Necas L, Hrubina M, Skotak M, Melisik M, Lisy P, Cibula Z. Bunionette deformity corrected with "shortening" scarf osteotomy of the fifth metatarsal: mid-term results of a 34-cases. Foot Ankle Surg 2019, http://dx.doi.org/10.1016/j.fas.2019.06.001.

[13] Michels F, Guillo S, Cordier G, Stevens N, Burssens A. Percutaneous bunionette correction: a cadaveric study. Clin Res Foot Ankle 2015;3:169.

[14] Giannini S, Faldini C, Vannini F, Digennaro V, Bevoni R, Luciani D. The minimally invasive osteotomy "SERI" (simple, effective, rapid, inexpensive) for correction of bunionette deformity. Foot Ankle Int 2008;29:282-6.

[15] Legenstein R, Bonomo J, Huber W, Boesch P. Correction of tailor's bunion with the Boesch technique: a retrospective study. Foot Ankle Int 2007:28:799-803.

[16] Magnan B, Samaila E, Merlini M, Bondi M, Mezzari S, Bartolozzi P. Percutaneous distal osteotomy of the fifth metatarsal for correction of bunionette. J Bone Joint Surg Am 2011;93:2116-22.

[17] Martinelli R, Valentini R. Correction of valgus of fifth metatarsal and varus of the fifth toes by percutaneous distal osteotomy. Foot Ankle Surg 2007;13:136-9.

[18] Waizy H, Olender G, Mansouri F, Floerkemeier T, Stukenborg-Colsman C. Minimally invasive osteotomy for symptomatic bunionette deformity is not advisable for severe deformities: a critical retrospective analysis of the results. Foot Ankle Spec 2012;5:91-6.

[19] Weitzel S, Trnka HJ, Petroutsas J. Transverse medial slide osteotomy for bunionette deformity: long-term results. Foot Ankle Int 2007;28:794-8.

[20] Lui TH. Percutaneous osteotomy of the fifth metatarsal for symptomatic bunionette. J Foot Ankle Surg 2014;53:747-52.

[21] Ceccarini P, Rinonapoli G, Nardi A, et al. Bunionette. Minimally invasive and percutaneous techniques: a topical review of literature. Foot Ankle Spec 2017;10:157-61.

[22] Shereff MJ, Yang QM, Kummer FJ, Frey CC, Greenidge N. Vascular anatomy of the fifth metatarsal. Foot Ankle 1991;11:350-3.

[23] Diebold PF. Basal osteotomy of the fifth metatarsal for the bunionette. Foot Ankle 1991;12:74-9.

[24] Tonogai I, Hayashi F, Tsuruo Y, Sairyo K. Direction and location of the nutrient artery to the fifth metatarsal at risk in osteotomy for bunionette. Foot Ankle Surg 2019;25:193-7 